Research Article

\title{
Difference in polypropylene fragmentation mechanism between marine and terrestrial regions
}

\author{
Hisayuki Nakatani ${ }^{1,2}$ (]) $\cdot$ Takuya Muraoka $^{1} \cdot$ Yuina Ohshima $^{1} \cdot$ Suguru Motokucho ${ }^{1,2}$
}

Received: 26 April 2021 / Accepted: 6 August 2021

Published online: 13 August 2021

(c) The Author(s) 2021 OPEN

\begin{abstract}
Two kinds of marine polypropylene (M1-PP and M2-PP) and one land PP (L-PP) samples were collected from two beaches and land in Japan, respectively, to study the fragmentation mechanisms. Delamination was observed on both M1-PP and M2-PP surfaces. Moreover, there was no delamination but an abrasion patch structure on the surface of L-PP. The delamination was studied using an advanced oxidation process-degraded PP as the marine PP model. The number and shape of cracks varied with an increase in degradation time. The fluctuations in the values and ratios of the carbonyl index as well as the weight change ratio were due to repeated oxidation and delamination. We found that the delamination behavior depends on the oxidation state. Poly(oxyethylene) ${ }_{8}$ octylphenyl ether (POE8) surfactant treatment caused the delamination to speed up, which is a typical characteristic of polyolefin environmental stress cracking (ESC). These results reveal that delamination is based on ESC.
\end{abstract}

\section{Article Highlights}

- Two kinds of marine and one land polypropylene (PP) samples were collected from two beaches and land, respectively, to study the fragmentation mechanisms.

- Delamination was observed on both of marine PP

- We found that the delamination was based on environmental stress cracking mechanism by employing a marine PP model. surfaces. Moreover, there was no delamination but an abrasion patch structure on the land PP surface.

Keywords Polypropylene · Marine · Land · Delamination · Environmental stress cracking

\section{Introduction}

Several plastic materials have yielded for a long time, resulting in the accumulation of a high amount of litter in the marine environment [1-15]. Plastic litter has become one of the major problems in the marine environment. In particular, microplastics (MP) regenerated by larger plastic debris have raised a growing environmental concern ([15-19]. Polypropylene (PP), polyethylene (PE), and expanded polystyrene (EPS) have low

Supplementary information The online version contains supplementary material available at https://doi.org/10.1007/s42452-021-047592.

$\triangle$ Hisayuki Nakatani, h-nakatani@nagasaki-u.ac.jp | ${ }^{1}$ Polymeri Materials Laboratory, Chemistry and Materials Program, Nagasaki University, 1-14 Bunkyo-machi, Nagasaki 852-8521, Japan. ${ }^{2}$ Organization for Marine Science and Technology, Nagasaki University, 1-14 Bunkyo-machi, Nagasaki 852-8521, Japan.

SN Applied Sciences ～～～（2021) 3:773 $\quad$ https://doi.org/10.1007/s42452-021-04759-2 
densities, and they are hugely produced worldwide. The MPs float on the sea surfaces. Besides, they are not biodegradable; hence, they semipermanently remain in the marine environment. The MP production place has not been clarified yet. For instance, one group has been generated in the terrestrial region (on land) and others in the sea. In some cases, MP partially leaves the sea to the atmosphere [20]. They occur in various places and circulate. Halle et al. reported the MP formation mechanism in the sea [9], however, the driving force for the fragmentation is not yet clarified. It has been reported that several kinds of MP are formed in water by light exposure in the visible and/or UV regions [21-23]. In our previous study, a PP film photodegradation test was performed in water with a specific photocatalyst under visible light irradiation, and MP particles were obtained by planar exfoliation [24]. We infer that MP formation is certainly associated with autoxidation and water. However, the detailed formation mechanism has not been clarified yet. It is unclear MPs are formed in the sea. The mechanism does not explain MP formation on dried terrestrial regions. The difference between the MP formation mechanism in marine and terrestrial regions is unknown. There is a need to clarify the difference in the MP formation mechanisms by studying the surfaces of weathered plastic debris in the sea and on land. The obtained hypothesis should be verified using an imitation model of the actual phenomenon.

In this study, two samples of marine PP and one land PP were collected to study the MP formation mechanism. The obtained mechanism was verified using an advanced oxidation process (AOP)-degraded PP as the marine PP model. The fragmentation mechanism of marine plastic litter has been presented for the first time in the world.

\section{Experiment}

\subsection{Materials}

PP (product name: J-700GP) was supplied by Prime Polymer Co., Ltd., Japan. The melt flow rate and density were $8 \mathrm{~g} / 10 \mathrm{~min}$ at $230{ }^{\circ} \mathrm{C}$ and $0.9 \mathrm{~g} / \mathrm{cm}^{3}$. Potassium hydroxide $(\mathrm{KOH})$ and potassium persulfate $\left(\mathrm{K}_{2} \mathrm{~S}_{2} \mathrm{O}_{8}\right)$ and poly(oxyethylene) ${ }_{8}$ octylphenyl ether (POE8) were purchased from Wako Pure Chemical Industries., Japan.

\subsection{PP sample collection}

Two kinds of marine PP litter pieces (M1-PP and M2-PP) were collected from Iguchihama beach at Tsushima island and Head land beach at Chigasaki city in Japan, respectively. One kind of terrestrial PP litter pieces (L-PP) was done from a dried island of Arakawa River at Tokyo in Japan. The detailed locations were shown in Fig. 1. A pyrolysis gas chromatography/mass (py-GC/MS: SHIMADZU GCMS-QP2010 PLUS, Japan) analysis was used to discriminate the PP litter pieces from other plastics. In Figure S1, the py-GC/MS chart of M1-PP was shown as representative results. All of them exhibited typical homo isotactic PP spectra. These pieces were pretreated using $\mathrm{KOH} 1 \mathrm{~N}$ aqueous solution.

\subsection{Advance oxidation process (AOP) degradation}

The PP was molded into a thin film $(50 \times 50 \times 0.065 \mathrm{~mm})$ by compression molding at $180^{\circ} \mathrm{C}$ under $10 \mathrm{MPa}$ for 10 $\mathrm{min}$, and then the film was cut to $10 \times 10 \times 0.065 \mathrm{~mm}$. In the case of heat pretreatment, the film was performed using an oven at $130^{\circ} \mathrm{C}$ for $144 \mathrm{~h}$ in air.
Fig. 1 Sampling locations of M1-PP , M2-PP and L-PP samples. Letters and numbers represent sampling sites, respectively. Local names and other information are given in Text

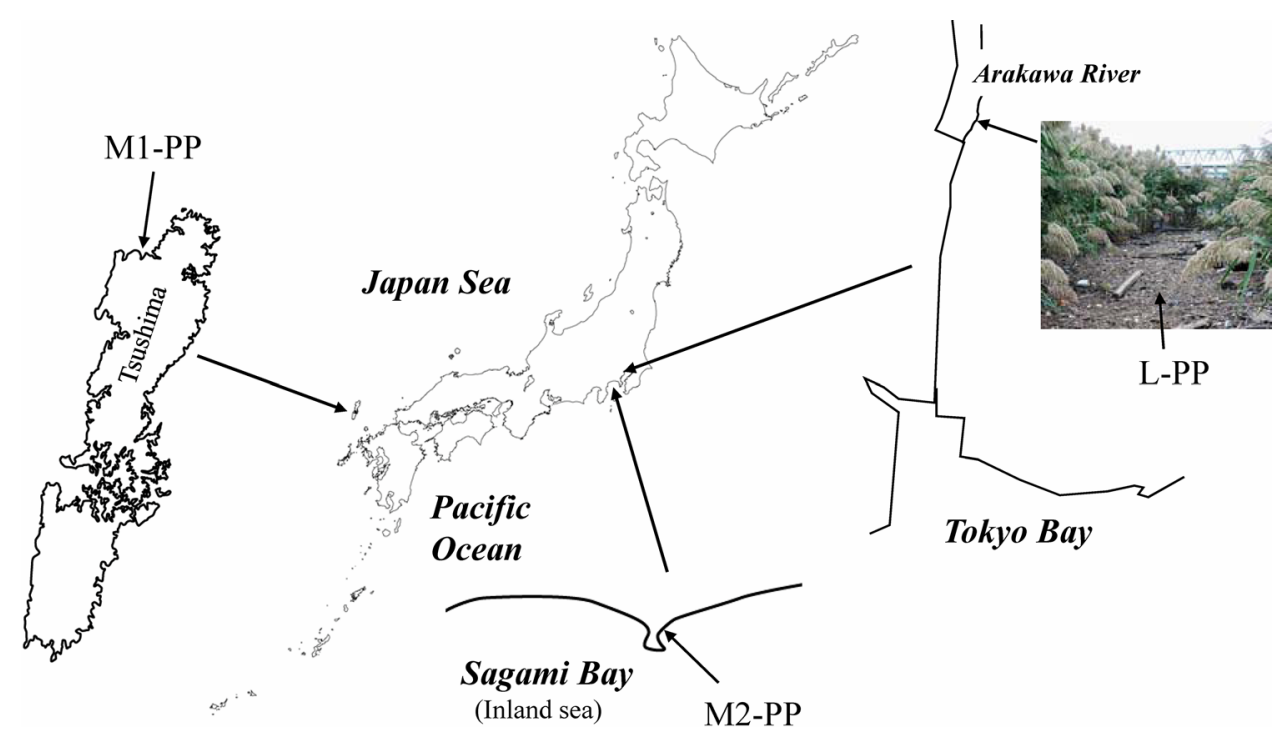


They were employed as "with heat pretreatment and without heat pretreatment" to a water oxidative degradation test, respectively. The AOP degradation procedure was according to some reports $[25,26]$. Each three pieces of the films were put into a $100 \mathrm{ml}$ glass vessel equipped with a 20 $\mathrm{ml}$ aqueous solution containing $0.54 \mathrm{~g} \mathrm{~K}_{2} \mathrm{~S}_{2} \mathrm{O}_{8}$ at ca. $65^{\circ} \mathrm{C}$ for $12 \mathrm{~h}$ under stirring with a stirrer tip speed of ca. $100 \mathrm{rpm}$, and the equal amount of $\mathrm{K}_{2} \mathrm{~S}_{2} \mathrm{O}_{8}$ aqueous solution was replaced every $12 \mathrm{~h}$ due to the consumption of the oxidant. The pieces were filtrated and were washed every 2 days to prevent the accumulation of $\mathrm{K}_{2} \mathrm{SO}_{4}$ in the solution and were again introduced into the fresh $\mathrm{K}_{2} \mathrm{~S}_{2} \mathrm{O}_{8}$ aqueous solution. The films were continuously treated according to the above procedures for different time periods. The oxidative degradation using $\mathrm{K}_{2} \mathrm{~S}_{2} \mathrm{O}_{8}$ was named as "AOP".

\subsection{Poly(oxyethylene) ${ }_{8}$ octylphenyl ether (POE8) surfactant treatment}

The POE8 surfactant treatment of the 9 days-AOP degraded sample with the heat pretreatment was performed using a $100 \mathrm{ml}$ glass vessel equipped with a $50 \mathrm{ml}$ aqueous solution containing 5 wt\%-POE8 at r.t. for 2 days.

\subsection{Weight measurement of PP and pre-PP films treated by water oxidative degradation using $\mathrm{K}_{2} \mathrm{~S}_{2} \mathrm{O}_{8}$}

The 3 pieces (ca. $15 \mathrm{mg}$ ) of the film at every one day were carefully picked up from the glass vessel with tweezers and were rinsed with methanol. After drying with vacuum oven at $60^{\circ} \mathrm{C}$ for $7 \mathrm{~h}$, the weight was measured.

\subsection{Characterization}

The morphology of PP sample surface was observed through SEM (JEOL SM-7500FAM, Japan), polarized optical microscope (POM: Nikon ECLIPSE 50/POL, Japan) and 3D digital microscope (Keyence VHX-7000, Japan). Then the 3D topological profile was subsequently evaluated on the Keyence VHX-7000.

The Fourier transform infrared (FT-IR) spectra were measured using an FT-IR spectrometer (Jasco IRT-5200, Japan) with an Attenuated Total Reflectance (ATR) diamond accessory at a resolution of $4 \mathrm{~cm}^{-1}$ over the full mid-IR range $\left(400-4000 \mathrm{~cm}^{-1}\right)$.

\section{Results and discussion}

Figure 2 shows the photograph, polarized optical microscope (POM) and microphotographs of M1-PP, M2-PP, and L-PP. M1-PP and M2-PP were collected from different beaches, and L-PP was collected from a place (see Fig. 1). The surface of M2-PP was too rough to be observed with $\mathrm{POM}$; it was observed using a microscope. Tile-like cracks were observed in the entire surface of M1-PP. Meanwhile, a rectangular crack pattern was observed on the L-PP surface. Crack growth causes fragmentation. The difference in the crack patterns suggests that the fragmentation mechanisms in the two samples are different. Several small flakes called "delamination" $[27,28]$ were observed on the M2-PP surface. Besides, the M2-PP surface showed a complicated structure composed of horizontal planes and protuberances, such as mirrors and hills. Figures S2 and S3 show the photographs of M2-PP. The 3D topological profiles show a step structure on the surfaces, which is attributed to multiple factors. Oxidative degradation is a dominant factor that induces fragmentation in PP [29]. However, a simple degradation model based on autoxidation cannot determine the formation mechanism of such a step structure. Figure 3 shows the SEM micrographs of M1-PP and L1-PP. Mirror planes and hills were distinctly observed in the SEM photograph of the M1-PP surface. The sampling locations are very far apart (over $1000 \mathrm{~km}$ ), and they face on the open-sea with high wave intensity and on inland-sea with weak wave intensity (Fig. 1), respectively. Various factors, such as voyage length, exposure history, and wave intensity to wave action, were different among the samples. The mirror plane area, hill magnitude, and delamination amount were dependent on the sampling location. For instance, M2-PP showed considerably rough surfaces compared with M1-PP. Moreover, step structures were observed on M1-PP and M2-PP, regardless of the sampling location. Weinstein et al. observed the delamination behavior in PP and PE degradation in a salt marsh $[27,28]$. The surface morphology of PP beach debris was also studied by Cooper et al., and they reported deep and defined fractures, indicating the occurrence of mechanical-related degradation [30]. The morphology of M1-PP(a) is similar to that reported by Cooper et al. We infer that the step structure is inherent in MP obtained in the sea and sea-like places. Waves in the sea continuously apply stresses on the debris, leading to environmental stress cracking (ESC). Delamination likely occurs in the sea due to ESC [31]. The morphology of the L-PP surface is considerably different from that of M1-PP, as shown in Fig. 3. It is composed of linear fractures and patches, and defined step structures were not observed. It implies that L-PP was exposed to common weathering without ESC. The L-PP fragmentation is attributed to weathering and surface abrasion by wind and other factors. These results suggest that there the fragmentation mechanism differs in marine and terrestrial regions.

The delamination mechanism is based on ESC. This hypothesis needs to be thoroughly tested using an 

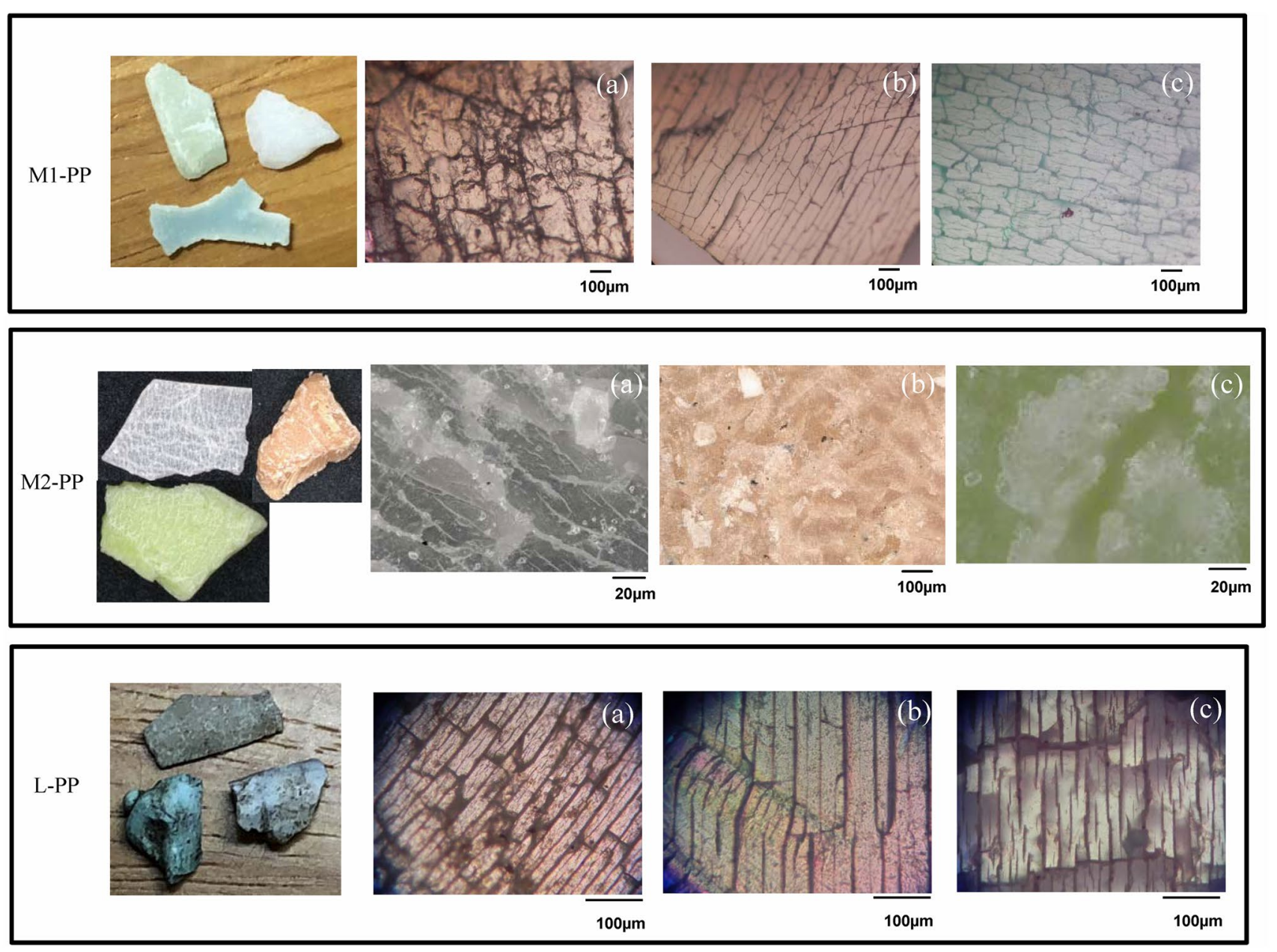

Fig. 2 Photographs, POM and microphotographs of M1-PP, M2-PP and L-PP. M1-PP and L-PP: POM. M2-PP: Microscope

imitation model of the actual phenomenon. PP degradation is initiated by autoxidation, as shown in Fig. 4 [32, 33], leading to the onset of ESC. The initiation rate of autoxidation is considerably slow under sunlight irradiation. Therefore, $\mathrm{K}_{2} \mathrm{~S}_{2} \mathrm{O}_{8}$ was employed as the initiator $[25,26]$. Highly reactive sulfate radicals are generated by cleaving the peroxide bond in $\mathrm{K}_{2} \mathrm{~S}_{2} \mathrm{O}_{8}$ and attacking PP chains. Autoxidation effectively advances, leading to the growth of cracks at the surface of the PP sample. The crack formation is related to the chemi-crystallization process, of which the driving force is autoxidation [34, 35]. Linear cracks named "linear fractures" are frequently observed on the surfaces of PP and PE debris $[30,36]$ and they were also observed on our PP debris samples. The cracks grow along flow-lines, i.e., the polymer chain orientation [34, 35]. The orientation generates a residual stress point to initiate cracks. An oriented surface part supplies linearly marked cracks, such as linear fracture, as shown in Figure S4(a). Moreover, the orientation degree depends on the depth direction of the sample. The surface has a higher orientation degree than the inner part. Therefore, the orientation becomes weaker as autoxidation advances to the inner part. The number and shape of cracks change with an increase in degradation time when ESC occurs on AOP-degraded PP as an imitation model of marine MP degradation. Longer AOPdegraded PP showed a mixture of tile- and rectangle-like crack patterns, as shown in Figure S4(b). The tile-like crack pattern represents crack growth around PP spherulites. In our previous work, we confirmed that the PP spherulite structure contributes to the tile-like crack pattern [24]. The occurrence of such a crack pattern is inherent in PP when the orientation does not exist in the matrix. Only the tile-like crack pattern was observed due to the release from the orientation by the heat pretreatment, as shown in Figure S4(c). Linear fracture is formed on the surface by orientation. However, it gradually changes to the tilelike crack pattern. The orientation degree becomes lower down the internal part due to the increase in delamination. FT-IR spectra of the AOP-degraded PP samples reveal that autoxidation, as well as typical weathering of $\mathrm{PP}$, occurred 
Fig. 3 SEM micrographs and plausible mechanisms to marine and land PP debris
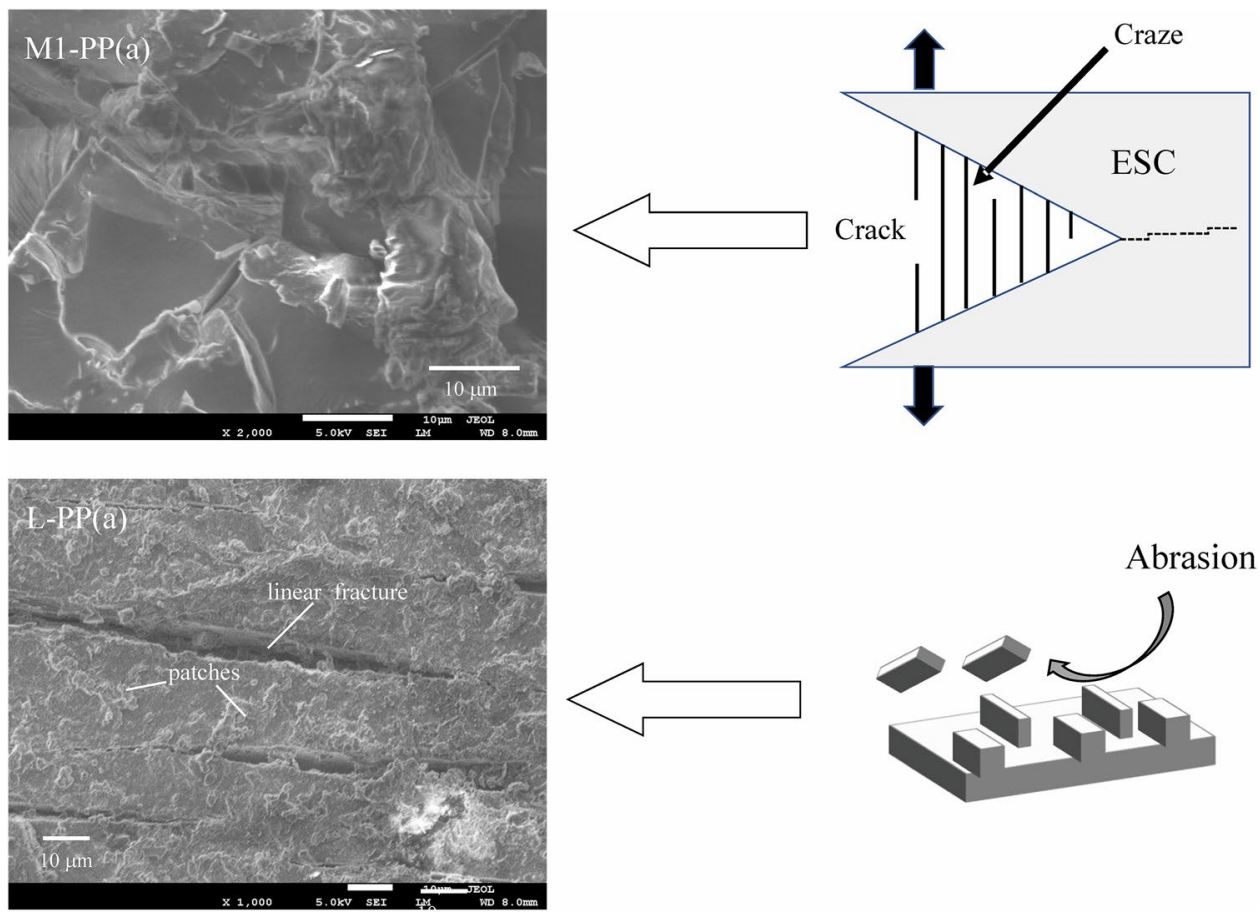

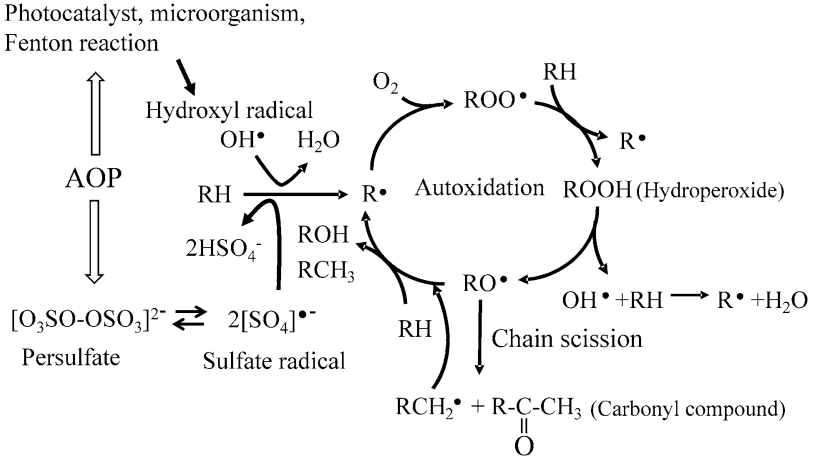

Fig. 4 Autoxidation mechanism initiated by advanced oxidation process (AOP)

(Figure S5). Figure 5 shows the value and change rate of carbonyl index $(\mathrm{Cl})$, ratio, and the rate of weight change vs AOP degradation time for PP films with and without heat pretreatment. $\mathrm{Cl}$ was calculated using the carbonyl group (ca. $1720 \mathrm{~cm}^{-1}$ )/methylene group (ca. $1452 \mathrm{~cm}^{-1}$ ) band intensity ratio [37-39]. The positive and negative values, as well as the ratios, are attributed to the repeated oxidation and delamination [24]. In particular, the values and ratios of the samples with no heat pretreatment rise up and down unlike those for heat-pretreated samples. The difference is a result of the initial oxidation degree. The initial $\mathrm{Cl}$ values were 0.20 and 0.14 with and without heat pretreatment. The heat treatment brings about oxidation and leads to initial delamination speedup. In addition, heatpretreated samples oxidize to the inside and change to a rather degraded medium than pristine PP. The delamination behavior substantially depends on the oxidation state. Interestingly, the $\mathrm{Cl}$ and rates of weight change of the sample without heat pretreatment show a simple oscillation to the degradation day. The less degraded PP, i.e., nearly homogeneous matrix, showed repetition motion, of which delamination is simply repeated. Moreover, the pretreated sample composed of heterogeneously degraded matrix exhibited irregular delamination behavior. The dependence on PP matrix conditions can be interpreted by a delamination mechanism based on ESC. Figure 6 shows the ESC mechanism of PP delamination. The onset of crack is initiated by autoxidation, and a series of delamination proceeds as follows: (I) water ingress, (II) craze birth and crack growth, and (III) delamination. These processes are repeated. The weight is increased by autoxidation during the (I) and (II) steps because delamination is not accompanied, and it decreases at (III). The simple oscillation of $\mathrm{Cl}$ and rates of weight change are observed by the cycle of (I), (II), and (III) steps, as shown in Fig. 6. The delamination of marine PP samples is initiated as well. Notably, delamination occurs by ESC. Also, the ESC of polyolefin, such as polyethylene, shows surfactant-induced fracture in water [40]. Thus, delamination is likely facilitated in a water solution of surfactant. To assess the effect of the surfactant, the delamination mechanism is clarified. Figure 7 shows the optical SEM micrograph and 3D image of nine-day AOPdegraded PP with heat pretreatment. As shown in Fig. 7(a), there were no delamination parts on the surface before the POE8 surfactant treatment. Although the trace was 

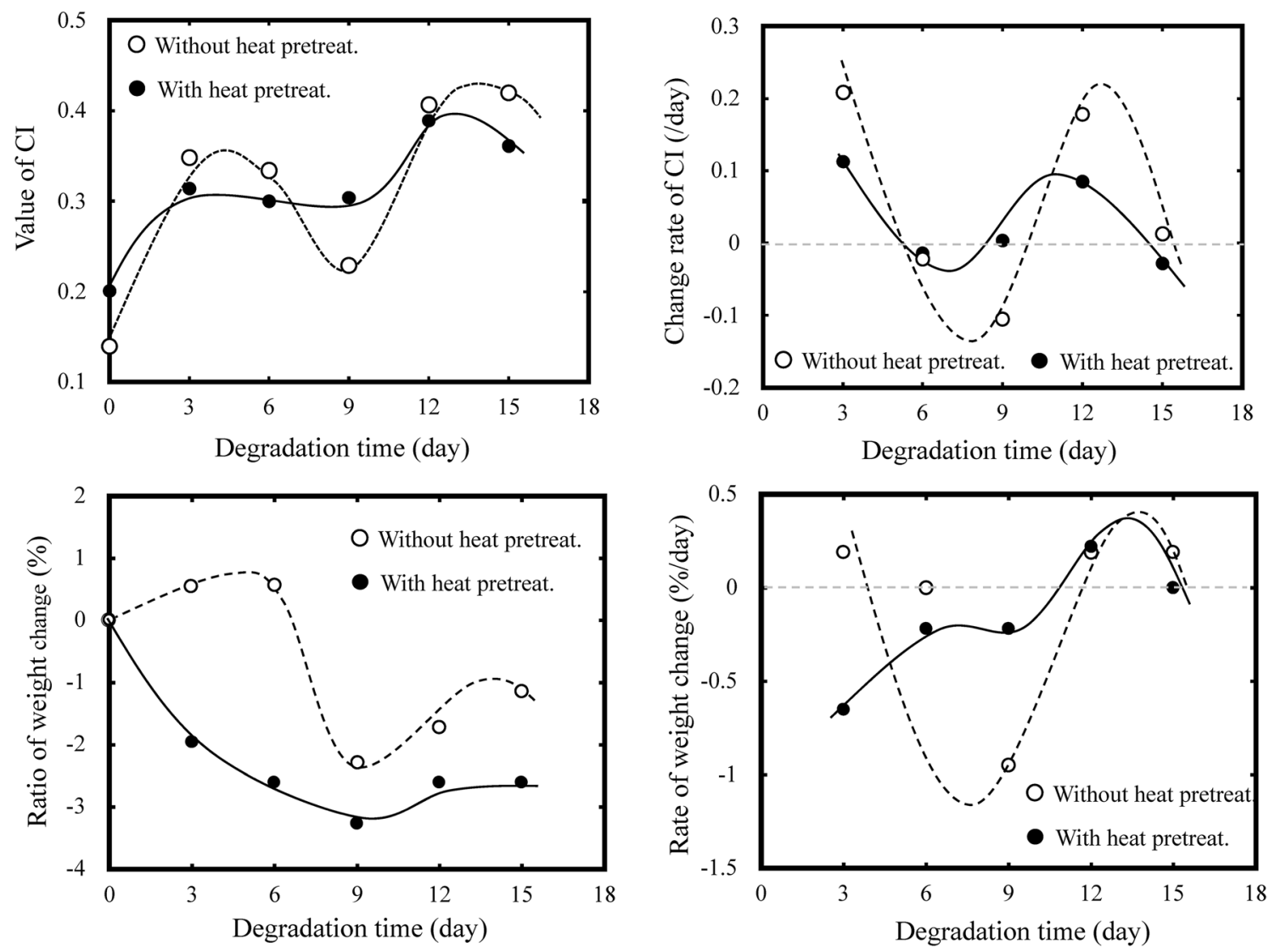

Fig. 5 Value and change rate of carbonyl index $(\mathrm{Cl})$, ratio and rate of weight change vs AOP degradation time of PP film with and without heat pretreatment

Fig. 6 ESC mechanism for PP delamination



(I)

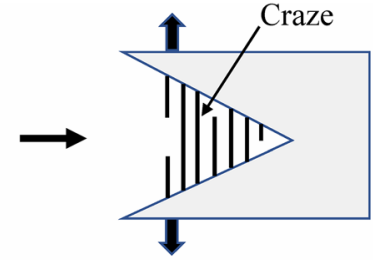

(II)

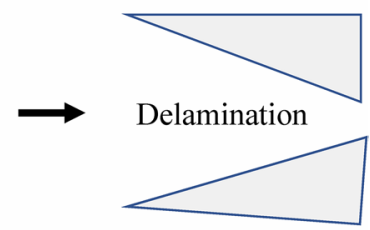

(III)



Degradation time 
Fig. 7 Optical SEM micrograph and 3D image of AOP degraded PP with heat pretreatment: AOP degradation time $=9$ days. a Before surfactant treatment. b After surfactant treatment
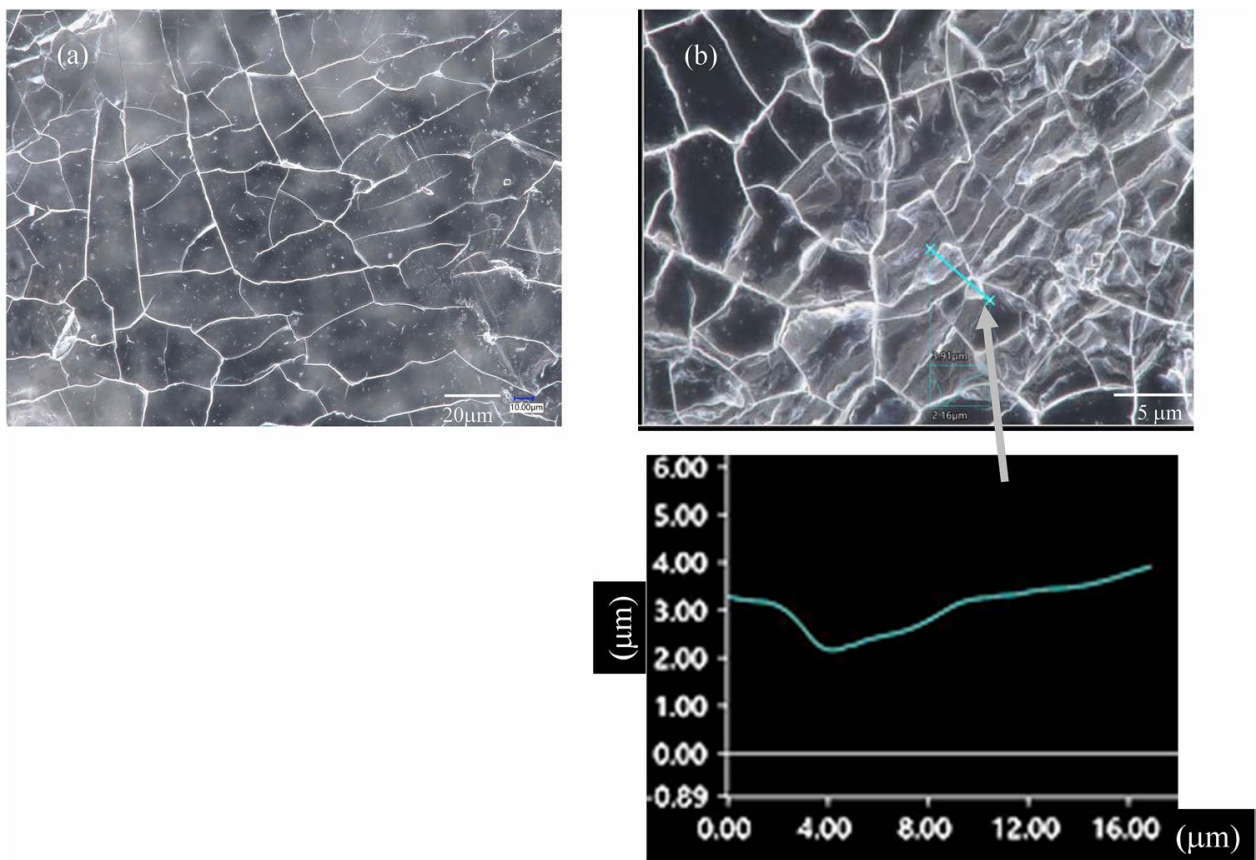

shallow (ca. $1 \mu \mathrm{m})$ and uneven, the surfactant treatment provided many delamination traces, which resulted in the speedup. The result shows that delamination is based on the ESC mechanism. In addition, the cross-section was considerably rough and not a mirror plane. The force of craze fibril breakdown was considerably weaker since the water turbulence intensity is lower than the sea waves. The roughness of the cross-section depends on the water turbulence intensity, e.g., wave strength. Other M1-PP samples showed rough cross-sections similar to that of the surfactant-treated sample (Figure S6). In addition, as shown in Figure S7, there are many delamination traces on the large area. The trace shapes reveal that tile-like peeling off occurs. These results suggest that the shape of the delaminated part is affected by factors, such as wave intensity and drifting history. The fragmentation behavior of PP degradation is generally not due to ESC but surface embrittlement. It is a well-known fact [41]. However, all of the degradation test have been performed under dry condition without water. The water plays an important role in the degradation process. Julienne et al. suggested that water worked as plasticizer in polyolefin degradation process $[22,23]$. In fact, we succeeded in recovering peeling off parts from AOP-degraded PP surface as shown in Figure $\mathrm{S} 8$. The recovery part composes of many sheet-like slices. If the fragmentation occurs by surface embrittlement, the powdery recovery is obtained. The sheet-like slices support that ESC of PP can occur in the water presence. The delamination behavior is attributed to the ESC mechanism, however, more detailed studies are required to clarify the delamination shape and production rate.

\section{Conclusions}

Two marine PP (M1-PP and M2-PP) and one land PP (L-PP) samples were collected from two beaches and the land, respectively, to study the fragmentation mechanisms. The delamination behavior was observed on the surfaces of both marine PP samples. Meanwhile, L-PP showed no delamination but abrasion patches on the surface. The delamination mechanism was studied using AOP-degraded PP as the marine PP model. The number and shape of cracks changed with the degradation time, showing the occurrence of ESC. The positive and negative $\mathrm{Cl}$ values, as well as the ratios of the weight change, are attributed to repeated oxidation and delamination. In particular, the values and ratios of the sample without heat pretreatment fluctuated up and down, unlike those of the heat-pretreated samples. This is because of the different initial degrees of oxidation. The delamination behavior was dependent on the oxidation state, suggesting that the mechanism is based on ESC. Moreover, the POE8 surfactant treatment caused delamination speedup, which is a typical characteristic of polyolefin ESC. These results reveal that delamination is based on the ESC mechanism.

Acknowledgements This work was supported by the Grant-in-Aid for Scientific Research (No. 20K05587) from Japan Society for the Promotion of Science and by financial supports of Nagasaki University organization for marine science and technology and for function enhancement program of research. The authors would like to thank Mr. Akira Kanda (Japan Broadcasting Corporation) for providing PP samples. This study made use of IR instrument in the Advanced Material Science Research Unit Sharing System of Nagasaki University. 
Author contributions $\mathrm{HN}-$ Conceptualization, Formal analysis and investigation, Writing - original draft preparation and editing, Supervision. TM-Formal analysis and investigation. YO-Formal analysis and investigation. SM-Formal analysis and investigation.

Funding The Grant-in-Aid for Scientific Research (No. 20K05587) from Japan Society for the Promotion of Science, by the 9th Long-range Research Initiative, No. 19_D08-01 from Japan Chemical Industry and by financial supports of Nagasaki University organization for marine science and technology and for function enhancement program of research.

Data availability Data can be shared under request.

\section{Declarations}

Conflict of interest The authors declare that they have no conflict of interest.

Open Access This article is licensed under a Creative Commons Attribution 4.0 International License, which permits use, sharing, adaptation, distribution and reproduction in any medium or format, as long as you give appropriate credit to the original author(s) and the source, provide a link to the Creative Commons licence, and indicate if changes were made. The images or other third party material in this article are included in the article's Creative Commons licence, unless indicated otherwise in a credit line to the material. If material is not included in the article's Creative Commons licence and your intended use is not permitted by statutory regulation or exceeds the permitted use, you will need to obtain permission directly from the copyright holder. To view a copy of this licence, visit http://creativecommons. org/licenses/by/4.0/.

\section{References}

1. Derraik JGB (2002) The pollution of the marine environment by plastic debris: a review. Mar Poll Bull 44(9):842-852. https://doi. org/10.1016/S0025-326X(02)00220-5

2. Barnes DKA, Galgani F, Thompson RC, Barlaz M (2009) Accumulation and fragmentation of plastic debris in global environments. Phil Trans R Soc B 364(1526):1985-1998. https://doi.org/10. 1098/rstb.2008.0205

3. Thompson RC, Swan SH, Moore CJ, vom Saal FS (2009) Our plastic age. Phil Trans R Soc B 364(1526):1973-1976. https://doi.org/ 10.1098/rstb.2009.0054

4. Law KL, Morét-Ferguson S, Maximenko NA, Proskurowski G, Peacock EE, Hafner J, Redd CM (2010) Plastic accumulation in the North Atlantic subtropical gyre. Science 329(5996):1185-1188. https://doi.org/10.1126/science.1192321

5. Cózar A, Echevarría F, González-Gordillo Jl, Irigoien X, Ubeda B, León SH, Palma AT, Navarro S, Garcia-de-Lomas J, Ruiz A, Fernandez-de-puelles ML, Duarte CM (2014) Plastic debris in the open ocean. P Natl Acad Sci USA 111(28):10239-10244. https://doi. org/10.1073/pnas.1314705111

6. Eriksen M, Lebreton LCM, Carson HS, Thiel M, Moore CJ, Borerro JC (2014) Plastic pollution in the world's oceans: more than 5 trillion plastic pieces weighing over 250,000 tons afloat at sea. PLoS One 9(12):e111913. https://doi.org/10.1371/journal.pone. 0111913

7. Law KL, Thompson RC (2014) Microplastics in the seas. Science 345(6193):144-145. https://doi.org/10.1126/science.1254065

8. Jambeck JR, Geyer R, Wilcox C, Siegler TR, Perryman M, Andrady A, Narayan R, Law KL (2015) Plastic waste inputs from land into the ocean. Science 347(6223):768-771. https://doi.org/10.1126/ science.1260352

9. Halle AT, Ladirat L, Gendre X, Goudouneche D, Pusineri C, Routaboul C, Tenailleau C, Duployer B, Perez E (2016) Understanding the fragmentation pattern of marine plastic debris. Environ Sci Technol 50(11):5668-5675. https://doi.org/10.1021/acs.est. 6b00594

10. Avio CG, Gorbi S, Regoli F (2017) Plastics and microplastics in the oceans: from emerging pollutants to emerged threat. Mar Environ Res 128:2-11. https://doi.org/10.1016/j.marenvres.2016. 05.012

11. Law KL (2017) Plastics in the marine environment. Annu Rev Mar Sci 9:205-229. https://doi.org/10.1146/annur ev-marine-010816-060409

12. Rummel CD, Jahnke A, Gorokhova E, Kühnel D, Schmitt-Jansen $M$ (2017) Impacts of biofilm formation on the fate and potential effects of microplastic in the aquatic environment. Environ Sci Technol Lett 4:258-267. https://doi.org/10.1021/acs.estle tt.7b00164

13. Yokota $\mathrm{K}$, Waterfield $\mathrm{H}$, Hastings $\mathrm{C}$, Davidson $\mathrm{E}$, Kwietniewski $\mathrm{E}$, Wells $B$ (2017) Finding the missing piece of the aquatic plastic pollution puzzle: interaction between primary producers and microplastics. Limnol Oceanogr Lett 2:91-104. https://doi.org/ 10.1002/lol2.10040

14. Michels J, Stippkugel A, Lenz M, Wirtz K, Engel A (2018) Rapid aggregation of biofilm-covered microplastics with marine biogenic particles. Proc R Soc B 285:1203-1211. https://doi. org/10.1098/rspb.2018.1203

15. Isobe A, Iwasaki S, Uchida K, Tokai T (2019) Abundance of nonconservative microplastics in the upper ocean from 1957 to 2066. Nat Commun 10(417):1-13. https://doi.org/10.1038/ s41467-019-08316-9

16. Andrady AL (2011) Microplastics in the marine environment. Mar Pollut Bull 62(8):1596-1605. https://doi.org/10.1016/j. marpolbul.2011.05.030

17. Koelmans AA, Kooi M, Law KL, Van Sebille E (2017) All is not lost, deriving a top-down mass budget of plastic at sea. Environ Res Lett 12:114028. https://doi.org/10.1088/1748-9326/ aa9500

18. Cai L, Wang J, Peng J, Wu Z, Tan X (2018) Observation of the degradation of three types of plastic pellets exposed to UV irradiation in three different environments. Sci Total Environ 628-629:740-747. https://doi.org/10.1016/j.scitotenv.2018.02. 079

19. Gigault J, Halle AT, Baudrimont M, Pascal PY, Gauffre F, Phi T-L, Hadri HE, Grassl B, Reynaud S (2018) Current opinion, What is a nanoplastic? Environ Pollut 235:1030-1034. https://doi.org/10. 1016/j.envpol.2018.01.024

20. Allen S, Allen D, Moss K, Le Roux G, Phoenix VR, Sonke JE (2020) Examination of the ocean as a source for atmospheric microplastics. PLoS ONE 15(5):e0232746. https://doi.org/10.1371/ journal.pone. 0232746

21. Lambert S, Wagner M (2016) Formation of microscopic particles during the degradation of different polymers. Chemosphere 161:510-517. https://doi.org/10.1016/j.chemosphere.2016.07. 042

22. Julienne F, Delorme N, Lagarde F (2019) From macroplastics to microplastics Role of water in the fragmentation of polyethylene. Chemosphere 236:124409. https://doi.org/10.1016/j. chemosphere.2019.124409

23. Julienne F, Lagarde F, Delorme N (2019) Influence of the crystalline structure on the fragmentation of weathered polyolefines. Polym Degrad Stab 170:109012. https://doi.org/10.1016/j.polym degradstab.2019.109012

24. Nakatani H, Kyan T, Muraoka T (2020) An effect of water presence on surface exfoliation of polypropylene film initiated by 
photodegradation. J Polym Environ 28(8):2219-2226. https:// doi.org/10.1007/s10924-020-01769-w

25. Liu P, Qian L, Wang H, Zhan X, Lu K, Gu C, Gao S (2019) New insights into the aging behavior of microplastics accelerated by advanced oxidation processes. Environ Sci Technol 53(7):35793588. https://doi.org/10.1021/acs.est.9b00493

26. Lee J, von Gunten U, Kim JH (2020) Persulfate-Based advanced oxidation: critical assessment of opportunities and roadblocks. Environ Sci Technol 54(6):3064-3081. https://doi.org/10.1021/ acs.est.9b07082

27. Weinstein JE, Crocker BK, Gray AD (2016) From macroplastic to microplastic: degradation of high-density polyethylene, polypropylene, and polystyrene in a salt marsh habitat. Environ Toxicol Chem 35:1632-1640. https://doi.org/10.1002/etc.3432

28. Weinstein JE, Dekle JL, Leads RR, Hunter RA (2020) Degradation of bio-based and biodegradable plastics in a salt marsh habitat: another potential source of microplastics in coastal waters. Mar Pollut Bull 160:111518. https://doi.org/10.1016/j.marpolbul. 2020.111518

29. Sun Y, Yuan J, Zhou T, Zhao Y, Yu F, Ma J (2020) Laboratory simulation of microplastics weathering and its adsorption behaviors in an aqueous environment: a systematic review. Environ Pollut 265:114864. https://doi.org/10.1016/j.envpol.2020.114864

30. Cooper DA, Corcoran PL (2010) Effects of mechanical and chemical processes on the degradation of plastic beach debris on the island of Kauai, Hawaii. Mar Pollut Bull 60(5):650-654. https:// doi.org/10.1016/j.marpolbul.2009.12.026

31. Enfrin M, Dumée LF, Lee J (2019) Nano/microplastics in water and wastewater treatment processes-Origin, impact and potential solutions. Water Res 161(15):621-638. https://doi.org/ 10.1016/j.watres.2019.06.049

32. Adams JH (1970) Analysis of the nonvolatile oxidation products of polypropylene Thermal oxidation. J Polym Sci A. 8(5):10771090. https://doi.org/10.1002/pol.1970.150080505

33. Audouin L, Gueguen V, Tcharkhtchi A, Verdu J (1995) “Close loop” mechanistic schemes for hydrocarbon polymer oxidation. J Polym Sci A 33(6):921-927. https://doi.org/10.1002/pola.1995. 080330605
34. Rabello MS, White JR (1997) The role of physical structure and morphology in the photodegradation behaviour of polypropylene. Polym Degrad Stab 56(1):55-73. https://doi.org/10.1016/ S0141-3910(96)00202-9

35. Fechine GJM, Demarquette NR (2008) Cracking formation on the surface of extruded photodegraded polypropylene plates. Polym Eng Sci 48(2):365-372. https://doi.org/10.1002/pen. 20958

36. Corcoran PL, Biesinger MC, Grifi M (2009) Plastics and beaches: a degrading relationship. Mar Pollut Bull 58(1):80-84. https://doi. org/10.1016/j.marpolbul.2008.08.022

37. Carlsson DJ, Wiles DM (1969) The Photodegradation of Polypropylene Films. III. Photolysis of Polypropylene Hydroperoxides. Macromolecules 6(2):597-606. https://doi.org/10.1021/ma600 12a007

38. Kato Y, Carlsson DJ, Wiles DM (1969) The photo-oxidation of polypropylene: some effects of molecular order. J Appl Polym Sci 13(7):1447-1458. https://doi.org/10.1002/app.1969.07013 0708

39. Canopoli L, Coulon F, Wagland ST (2020) Degradation of excavated polyethylene and polypropylene waste from landfill. Sci Total Environ 698:134125. https://doi.org/10.1016/j.scitotenv. 2019.134125

40. Robeson LM (2013) Environmental stress cracking: a review. Polym Eng Sci 53(3):453-467. https://doi.org/10.1002/pen. 23284

41. Schoolenberg GE (1988) A fracture mechanics approach to the effects of UV-degradation on polypropylene. J Mater Sci 23:1580-1590. https://doi.org/10.1007/BF01115695

Publisher's Note Springer Nature remains neutral with regard to jurisdictional claims in published maps and institutional affiliations. 